\title{
LAS RADIOS UNIVERSITARIAS DE MÉXICO Y SUS ESTRATEGIAS PARA COMUNICAR LA CIENCIA EN INTERNET
}

\section{The University Radios of Mexico and its Strategies to Communicate Science on the Internet}

\section{As rádios universitárias do México e suas estratégias para comunicar a ciência na internet}

\section{Marina Vázquez Guerrero, Universidad de Colima (México)}

marina@ucol.mx

\section{Recibido: 16 de febrero de 2018}

Aprobado: 14 de mayo de 2018

\section{RESUMEN}

El presente trabajo muestra un análisis de las estrategias que tienen para comunicarse con su audiencia las radios universitarias de México y evidencia el nivel de incorporación de la web 2.0 a este tipo de medios de corte social y cultural que suman alrededor de 75 en todo el país. En particular se hace una revisión a los programas de comunicación pública de la ciencia y tecnología (PCST), desde sus formatos, creatividad y forma de distribución, que implican la posibilidad de que un usuario lo comparta o escuche a su tiempo y ritmo. Se recurrió para ello a la observación sistemática de cada uno de los sitios web de las emisoras, así como al análisis de contenido de las radios que mantienen en línea productos que divulgan la ciencia. Como resultados observamos que tan solo el $14 \%$ ofrece esta posibilidad, 
y el uso del podcast que tiene ventajas sobre otros, como la sindicación, el almacenamiento y la descarga en dispositivos móviles, se limita a seis casos, cuando significa una de las principales formas de difusión de contenidos, aliado importante de la radio actual.

Palabras clave: comunicación pública de la ciencia, radio universitaria, sonido, podcast.

\section{ABSTRACT}

This paper analyses the strategies used by Mexican university radio stations to communicate with their audience, particularly those showing some degree of integration of the web 2.0 to social and cultural media, which constitute around $75 \%$ of all media in the country. In particular, the article shows the analysis made of programs of public communication of science and technology focusing on format, creativity, and distribution techniques, which could foster the users' possibility to share and listen at his/her own pace. Exploration was done using systematic observation for exploring websites of radio stations as well as content analysis of scientific divulgation products broadcasted online. Results show that only $14 \%$ of radio stations offer this option and the use of podcast that has advantages over others such as syndication, storage, and downloading in mobile devices is limited to six cases. One of the main dissemination forms of contents and an essential ally of the current radio is podcasts.

Keywords: Public communication of science and technology, university radio, sound, podcast.

\section{RESUMO}

O presente trabalho mostra uma análise das estratégias que têm para comunicar-se com sua audiência as rádios universitárias do México e evidencia o nível de incorporação da web 2.0 a este tipo de meios de corte social e cultural que sumam ao redor de 75 em todo o país. Particularmente, se faz uma revisão aos programas de comunicação pública da ciência e tecnologia (PCST), desde seus formatos, criatividade e forma de distribuição que implicam a possibilidade de que um usuário o partilhe ou ouça a seu tempo e ritmo. Recorreu-se para isto À observação sistemática de cada um dos sites web das estações de rádio, assim como à análise de conteúdo das rádios que mantém uma linha, produtos que divulgam a ciência. Como resultados observamos que só o $14 \%$ oferecem a possibilidade e o uso do podcast que tem vantagens sobre outros, como a associação, o armazenamento, e a descarga em dispositivos móveis, se limita a seis casos, quando significa uma das principais formas de difusão de conteúdos, aliado importante da rádio atual.

Palavras-chave: comunicação pública da ciência, rádio universitária, som, podcast. 


\section{DISERTACIONES}

ESTUDIOS

\section{Introducción}

La radio ha sido uno de los medios masivos que se incorporó de manera un poco más tardía al uso de recursos en internet y, sin embargo, al día de hoy significa una alianza indispensable, la mejor forma de conectar con nuevas audiencias y de conservarlas, dado el crecimiento del consumo de la red de redes en el mundo. Muchas de las radios universitarias de México nacieron precisamente en la era analógica y durante mucho tiempo la señal por antena ha sido su prioridad. Las primeras emisoras de este tipo - que se caracterizan por ser no comerciales y dedicadas a la difusión de la educación, cultura y la ciencia- que comenzaron a transmitir su señal por internet lo hicieron alrededor del año 2002.

Estudios previos han demostrado (Berlín, 2000; Vázquez Guerrero, 2012) la diversidad de emisoras universitarias que existen en un país con más de 129 millones de habitantes, el décimo más poblado del mundo (Excélsior, 2017), y que generalizando se dividen en tres grandes grupos: las de universidades públicas, las de universidades privadas y las de institutos tecnológicos, siendo las primeras las más numerosas y principalmente con señal por AM O FM; las segundas con señal por internet, Fm y digital; y las terceras, que comparten señal por internet o fm y que han logrado un menor desarrollo que las anteriores.

La integración a la era digital ha sido paulatina y lenta en algunos casos, como se verá en los resultados de este estudio, donde se muestra la forma de incorporación a la web 2.0 de este tipo de medios de corte social y cultural que suman alrededor de 75 en todo el país.

Observando recursos que utilizan estas radios para compartir audio encontramos diferentes plataformas de 'radio a la carta', en algunos casos anunciada como podcast, pero que no todas permiten la descarga. Las plataformas de distribución de audio varían desde sencillos reproductores en línea hasta iTunes.

En particular se hace una revisión a las producciones de ciencia y las características que presentan en estos formatos, pensando en la divulgación y en la posibilidad de que un usuario las escuche y comparta a su tiempo y ritmo.

\section{La ciencia y la necesidad de cercanía hacia al ciudadano}

El conocimiento científico que se va generando en los diferentes ámbitos, pero principalmente en el público, requiere de darse a conocer a la sociedad para contribuir tanto en su formación como en su mejor comprensión de los fenómenos. Se sabe que este tipo de conocimiento solo se adquiere en un $5 \%$ en la educación formal y el resto "se acumula a lo largo de los años de vida por la vía informal, desde lo que asimilamos sentados delante de la televisión a lo que aprendemos en una visita a un museo" (De Semir, 2016, p. 52), de allí la relevancia de utilizar diferentes medios para que esta información llegue a los ciudadanos.

En Estados Unidos ha sido una labor constante desde los años ochenta, en los que se lanzó el proyecto "Science for all Americans" con recomendaciones para promover la alfabetización de todos los habitantes del país, al identificar que la gente tenía una serie de creencias erróneas sobre temas de su vida cotidiana (Orozco, 2016). 


\section{DISERTACIONES}

ESTUDIOS

El papel del sonido en la comunicación: contribución, funciones y efectos

ISSN: $1856-9536$

Doi: http://dx.doi.org/10.12804/revistas.urosario.edu.co/disertaciones/a.6550

Volumen 12, Número 2 / Julio-diciembre 2019

Versión PDF para imprimir desde

http://revistas.urosario.edu.co/index.php/disertaciones

De acuerdo con expertos en temas de comunicación, la ciencia no debe ser vista como algo separado de la gente, es cuestión social, pues "las aplicaciones de la ciencia inciden en nuestra vida diaria. Y la necesidad de comprenderla es legítima” (Calvo, 2006, p. 226).

Existen diferentes conceptos relacionados con la labor de dar a conocer la ciencia a la sociedad, entre ellos: periodismo científico, divulgación de la ciencia, alfabetización científica, popularización de la ciencia o comunicación social de la ciencia; no obstante, para este estudio nos enfocamos en la comunicación pública de la ciencia y las tecnologías (PCST) que, conforme con Fayard, tiene como fin "poner al alcance de la sociedad los cambios que se derivan de la evolución del papel de la ciencia y la tecnología" (2003, párr. 1), e incluye en sus funciones a la divulgación, con nuevos elementos. "Uno de los más fundamentales es que toma en cuenta el punto de vista y las representaciones particulares de los no especialistas a los que se dirige” (Fayard, 2005, p. 42).

Las universidades están comprometidas en la divulgación de sus conocimientos y es necesario poner al alcance de la mayoría lo que va produciendo la humanidad. Como afirma Muñoz (2008, p. 240), es importante hacerlo "dándole el sentido y la responsabilidad ética y política, necesitamos información, tecnología y ciencia, con conciencia".

Cumplir con esta labor no es sencillo, pues, primero, las personas no siempre están en la disposición de aprender, de ser educados, pero tienen curiosidad y "un interés pragmático por aquellos resultados científicos directamente relacionados con sus necesidades cotidianas” (Alcíbar, 2015, p. 8), que deben ser tomados en cuenta para crear productos de divulgación donde no se abuse del lenguaje técnico y se eviten la solemnidad y el aburrimiento. Aunque las personas afirmen que la ciencia es importante, no se refleja ese interés en los museos o espacios dedicados para ello, el trabajo desde los medios de comunicación sigue siendo muy pertinente porque el contenido aparece dentro de su consumo cotidiano.

\section{La comunicación de la ciencia y sus limitaciones en México}

México es un país donde se destinan pocos recursos para las áreas de investigación y desarrollo, con tan solo un 0,5\% de su producto interno bruto (PIB), según estudios realizados por la Unesco (2015). El incremento presupuestal a la ciencia es un reclamo constante de la comunidad científica, sin embargo, esto no debe limitar en ellos la labor de interesar a los ciudadanos por la cultura científica.

Es necesario revertir las creencias de los mexicanos, ya que, de acuerdo con la Encuesta sobre la Percepción Pública de la Ciencia y la Tecnología (Inegi, 2011), un 38\% de las personas 'sin instrucción' cree que la ciencia es aburrida. Otros estudios (Polino \& Castelfranchi, 2017) confirman que alrededor del $70 \%$ de los mexicanos hace un consumo bajo de información científica. Ante la pregunta de ¿escucha secciones o programas de radio que tratan sobre ciencia y tecnología?, que los mismos autores hicieron a nivel Iberoamérica, la mayoría de personas responde "No, casi nunca o nunca". Y es que la preferencia a nivel general es por la televisión y de manera más creciente por internet.

El Consejo Nacional de Ciencia y Tecnología (Conacyt) ha tomado medidas al respecto y desde hace algunos años incrementó el apoyo para esta labor, promoviendo entre todos sus beneficiarios el ejercicio de la comunicación de sus proyectos inéditos, pero los frutos tardarán en ser valorados por la totalidad de los destinatarios.

\section{3}




\section{DISERTACIONES}

ESTUDIOS

El papel del sonido en la comunicación: contribución, funciones y efectos

ISSN: $1856-9536$

Doi: http://dx.doi.org/10.12804/revistas.urosario.edu.co/disertaciones/a.6550

Volumen 12, Número 2 / Julio-diciembre 2019

Versión PDF para imprimir desde

http://revistas.urosario.edu.co/index.php/disertaciones

Por otra parte, conforme con un estudio que hizo la empresa de opinión Parametría (2016) para el grupo radiofónico Imer de México, los temas que más les gusta escuchar a su público son, en primer lugar, sobre educación y artes, y divulgación científica y nuevas tecnologías con el $68 \%$, seguido de periodismo de investigación con el $60 \%$, es decir que el tema interesa a las personas y ellas mismas identifican a las emisoras no comerciales como las ideales para ofrecer este tipo de información de manera confiable.

\section{La radio y su labor formativa}

En lo que respecta al uso de la radio y el sonido con fines educativos, formativos y de divulgación del conocimiento, fue una práctica pionera del medio que se fue perdiendo con el paso del tiempo y el desarrollo como empresa comercial. Las primeras emisoras que se establecieron en los años veinte y que nacieron en las universidades de Estados Unidos se dedicaron a la enseñanza de la música y el arte para los granjeros y sus familias, con el fin de elevar su nivel social y cultural (Slotten, 2006).

En América Latina existió Radio Sutatenza, que "se dedicó durante casi 50 años a impartir lecciones educativas a campesinos sin formación" (Rodero, et al., 2014, p. 415), una experiencia que se replicó con éxito en Chile, Argentina, Perú, Bolivia, Venezuela, Nicaragua y España con Radio ECCA, en las islas Canarias.

En México tampoco son ajenas estas prácticas, pues en 1924 se fundó Radio Educación, primera emisora cultural que, si bien tuvo transmisiones irregulares durante más de 30 años por situaciones de tipo administrativo y político (Radio Educación, 2018), actualmente es una radio pública con la figura legal de órgano desconcentrado de la Secretaría de Cultura del gobierno federal, que emite contenidos culturales, educativos y artísticos de calidad, incursionando con acierto en internet con un portal alternativo a la señal en directo denominado E-radio (https:// www.e-radio.edu.mx/Inicio), el cual permite escuchar podcasts y armar tu propia radio seleccionando contenidos, y mantiene la comunicación con su audiencia a través de las redes sociales.

A fines de los años setenta también comenzaron a otorgarse permisos de radio en México para grupos minoritarios y así nacen las emisoras indigenistas, comunitarias, educativas, culturales y experimentales (Vázquez Guerrero, 2012). A partir de 1983, el gobierno crea el Instituto Mexicano de la Radio (Imer), que forma parte de la Secretaría de Educación Pública. El medio público tiene actualmente 18 emisoras y 39 canales digitales, y las principales temáticas que presenta son relacionadas con la salud, orientación, sociedad y cultura, ciencia y tecnología, política y economía, musicales e infantiles, según los cinco ejes temáticos del Plan Nacional de Desarrollo 2013-2018 (Imer, 2018). Su incursión a la era 2.0 ha sido lenta, pues aunque todas las emisoras ofrecen señal en streaming, la única opción que presenta, de radio diferida o a la carta, es una sección de Fonoteca sin posibilidad de descarga.

En el país también existe un grupo numeroso de 56 sistemas de radio y televisión públicas, perteneciente a los gobiernos de los Estados y que se agrupan en la Red de Radiodifusoras y Televisoras Educativas y Culturales de México, A. C., desde 2005. Las principales temáticas que difunde son de corte cultural, entre las que se incluyen contenidos sobre ciencia y tecnología. 


\section{DISERTACIONES}

ESTUDIOS

\section{La radio universitaria y la ciencia}

Las universidades a partir del siglo xix tienen como propósito abrirse a la sociedad y difundir el conocimiento. La extensión universitaria sigue siendo desde esa época una de sus funciones más relevantes, planteada en sus inicios con el objetivo de "irradiar los bienes de la cultura universitaria a las masas" (Rodríguez, 2004, p. 2), y que posteriormente se fue proyectando en otros términos más sociales y educativos, como refieren las misiones de muchas macrouniversidades actuales en América Latina, donde buscan "difundir la ciencia y la cultura bajo el principio de libertad y compromiso social, para responder a los problemas de su entorno regional y nacional" (Didriksson, 2008, p. 430).

En la universidad se propicia la investigación científica y a través de la extensión se da la posibilidad de difundirla. La mayoría de las radios universitarias dependen precisamente de esta área dentro del organigrama institucional y tienen, por lo tanto, esa función. No obstante, no en todos los casos es posible escuchar este tipo de contenidos.

Uno de los casos más notables se observa en la radio de la Universidad Nacional Autónoma de México, la cual mantiene al aire producciones de alta calidad desde los años ochenta, fecha en que se creó la Dirección General de Divulgación de la Ciencia (DGDC-UnAm). La emisora trabaja muy de la mano de este departamento, que cuenta con divulgadores especializados que realizan producciones y actividades de divulgación para su transmisión por medios de comunicación (Tagüeña, Rojas \& Reynoso, 2006).

En un estudio previo, efectuado en 2015, se observaba que eran 23 las radios mexicanas que reportaban 74 diferentes producciones en sus cartas programáticas sin tomar en cuenta los espacios informativos donde se incluyen notas cortas, lo que significa un promedio de 1,85 programas por emisora. Las emisoras con mayor producción de programas de temas científicos eran Radio Universidad Autónoma de Sinaloa con 12 productos y la Radio de la Universidad de Ciencias y Artes de Chiapas con 10; sin embargo, de ese total solamente 11 instituciones mantenían disponibles en su página web estas producciones para ser escuchadas a demanda (Vázquez Guerrero, Martín Pena \& Parejo, 2015).

Los temas más promovidos por estas radios incluyen la medicina, economía, antropología, ecología, biología, arquitectura y diseño, química, biotecnología, historia, neurobiología, turismo y astronomía, la mayoría de tipo magazine o revista semanal, con duración de entre 30 a 60 minutos, "donde se ven involucrados diferentes centros de investigación de las instituciones [...] o con la participación de departamentos de estudios o facultades interesados en dar a conocer su trabajo" (Vázquez Guerrero, et al., 2015, p. 15). Esto refleja, sin duda, que sí existe una producción importante de material de divulgación de la ciencia en las radios universitarias, pero se aprovecha muy poco al no abrir la posibilidad del audio a la carta.

\section{La radio en la era de internet}

La era 2.0, que consistió en la posibilidad de que el propio usuario creara páginas y contenidos compartidos en internet, y que se estima comenzó hacia 2004, trajo también a la radio una importante novedad con el podcast. Este formato sonoro, que puede realizar cualquier persona con su equipo casero o una radio, con la calidad broadcasting, rompió con lo efímero del medio para convertirlo en un producto coleccionable, para descargar y 


\section{DISERTACIONES}

ESTUDIOS

El papel del sonido en la comunicación: contribución, funciones y efectos

ISSN: 1856-9536

Doi: http://dx.doi.org/10.12804/revistas.urosario.edu.co/disertaciones/a.6550

Volumen 12, Número 2 / Julio-diciembre 2019

Versión PDF para imprimir desde

http://revistas.urosario.edu.co/index.php/disertaciones

escuchar cuando el usuario lo decida, generalmente en un reproductor portátil. Si bien en nuestro país no se ha generalizado el consumo cotidiano del podcast, existe un fuerte movimiento de profesionales y jóvenes que hacen buen uso de él, distribuyendo y promoviendo con apoyo de las redes sociales.

Por otra parte, la radio tiene la amplia posibilidad de hacer fusiones o cross-media, "que combinan soluciones de producción-realización gráfica, web, video digital y sonido” (Gertrudix \& García García, 2011, p. 253) y que permiten no solo escuchar, sino también 'conocer', con el apoyo de la imagen y texto. Ante la cada vez más limitada plantilla de una radio, quien domine la parte audiovisual, digital y tecnológica, tendrá trabajo porque existe una "demanda in crescendo de formación especializada [...] - que no creativa- que centra hoy en día el llamado reciclaje profesional en el mundo de la comunicación” (López Vidales, 2011, p. 32).

En México este acercamiento ha sido limitado. Hasta hace muy poco tiempo las empresas vieron la necesidad de interactuar de otra manera con sus audiencias y ofrecer dinámicas más juveniles. Ahora queda ver cómo se han ido integrando en particular al caso que nos ocupa: las radios universitarias.

\section{Metodología}

El objetivo de este texto es analizar las estrategias para comunicar la ciencia a través de internet de las radios universitarias mexicanas. Se recurrió para ello a la observación sistemática de los 75 sitios web oficiales de emisoras universitarias mexicanas, revisando las categorías de: sitio web, señal online, uso de podcast o similar, Facebook, Twitter, Instagram, YouTube, blog y otras. Se descartaron algunas redes sociales que se anunciaban como propias y pertenecían a la institución educativa. Se contabilizaron, no obstante, las que tenían tiempo sin actividad.

Posteriormente, se aplicó la observación a detalle de la programación de las 28 emisoras que hacían uso del podcast o plataformas de almacenamiento y distribución online. Una vez localizadas las radios que mantienen en línea productos para divulgar la ciencia y, por lo tanto, hacer comunicación pública, se realizó el análisis de contenido.

La muestra final está conformada por 11 emisoras que emiten 28 producciones, representando tan solo el $14 \%$ de las existentes (tabla 1). Dos de ellas son emisoras de universidades privadas, 1 del Instituto Tecnológico y el resto de universidades públicas.

Tabla 1. Emisoras universitarias de México que realizan comunicación pública de la ciencia online

\begin{tabular}{|l|l|}
\hline \multicolumn{2}{|c|}{ Emisoras y producciones } \\
\hline Concepto Radial: 2 & Instituto Tecnológico de Celaya: 1 \\
\hline Radio UABC: 5 & Radio UADY: 1 \\
\hline Radio UAm: 5 & Radio UdeG Autlán: 1 \\
\hline Radio UdeG Gdl.: 2 & Radio UdeG Vallarta: 1 \\
\hline Radio UnAm: 8 & Radio UdeM: 1 \\
\hline UAQro: 1 & \\
\hline
\end{tabular}

* La temporalidad del estudio abarca de agosto de 2017 a febrero de 2018.

Fuente: elaboración de la autora. 


\section{Resultados}

En la primera etapa de observación sistemática de sitios web, se identificaron todas las herramientas de internet que utilizan las radios para comunicarse con sus públicos. Aquí es posible observar que, a pesar de los años de acceso a la red en el país, aún existen emisoras que no cuentan con una página web básica donde la audiencia pueda consultar por lo menos su programación. Se trata del $14 \%$ (11 radios), que en su mayoría pertenece a institutos tecnológicos. Sin embargo, tres de estas emisoras manejan toda su información a través de su página de Facebook, que es la herramienta más común que se utiliza en esta revisión de infraestructura digital.

Como se observa en la figura 1, la red social Facebook ha superado en número a las páginas web, destacando que quienes no la tienen siguen siendo en su mayoría institutos tecnológicos, la Universidad Autónoma de Querétaro y la Universidad Pedagógica Nacional.

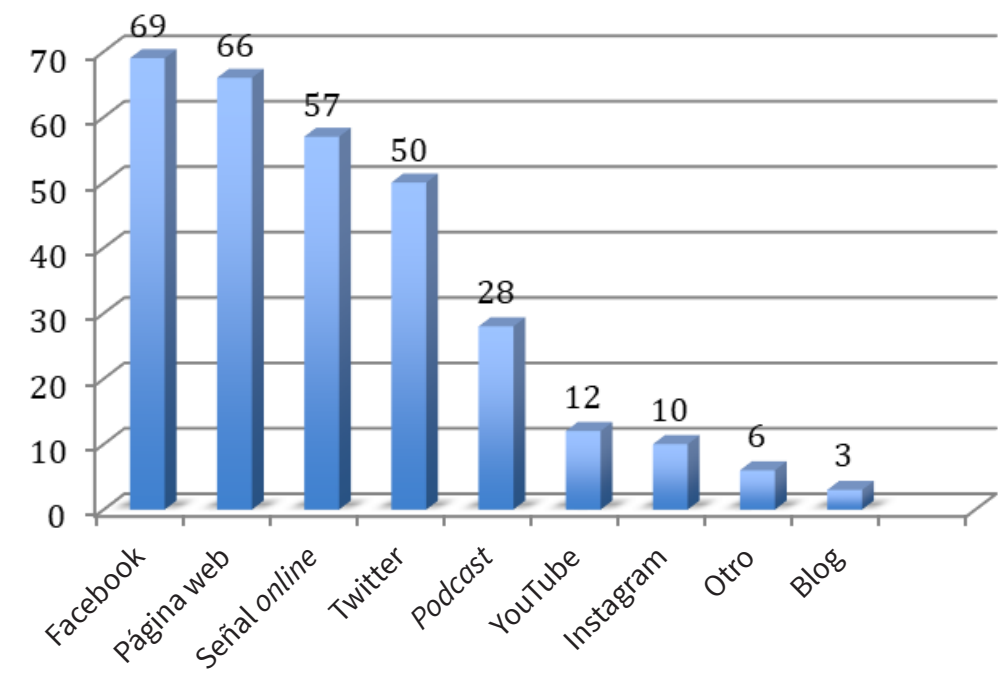

Figura 1. Uso de herramientas de internet en radios universitarias Fuente: elaboración de la autora.

La transmisión en streaming se ha ido generalizando en las radios universitarias. En algunos casos resulta ser la única opción, no obstante, el 26 \% (20) aún no lo practican, cuando existen muchos recursos técnicos para hacerlo y ampliar geográficamente sus escuchas.

Twitter, microblog que permite en pocos caracteres emitir un mensaje o enlazar a un sitio web, es utilizado por 50 radios, que representan un $65 \%$. Respecto al uso de YouTube, otra red social que se ha convertido en una de las más utilizadas por la gente joven, solamente 12 emisoras (16\%) cuentan con un canal. Destacan entre ellas la Autónoma de Chapingo, la Universidad Autónoma de Ciudad Juárez, Universo 94.9 de Colima, Concepto Radial del ITESm campus Ciudad de México, Radio Anáhuac, Radio uam, Ibero 90.9, Radio unam y unı Radio del Estado de México. 


\section{DISERTACIONES}

ESTUDIOS

La red social Instagram, que también en México tiene un número creciente de seguidores, es utilizada solo en 10 (13\%), donde coinciden varias radios que usan la anterior red -Twitter-, con la inclusión de la radio de la Universidad Autónoma del Carmen y unes de Durango.

El blog, que fue durante mucho tiempo una opción para publicar temas sin la necesidad de un gestor de página web, solo lo utilizan las radios Universo 94.9, la Universidad Autónoma de Yucatán (UADY) y la Autónoma de Campeche. Entre las demás herramientas existentes con las que trabajan algunas radios universitarias son Pinterest, Google+, aplicaciones para celular y listas en Spotify, en cinco radios.

\section{Plataformas de almacenamiento y reproducción de audio}

En la radio universitaria mexicana existen 28 emisoras (36\%) que hacen uso de alguna plataforma de almacenamiento y reproducción de audio para ser consultado fuera de los horarios de transmisión anunciados en su carta programática. En algunos casos lo generalizan como podcast y en otros, como audio a la carta. Las plataformas de distribución de audio que utilizan varían desde un sencillo reproductor de sonido, MediaFire, Podbean, Mixcloud, Soundcloud, iTunes e iVoox.

Haciendo la revisión detallada de las páginas web de estas emisoras, detectamos que el podcast, que significa una de las principales formas de difusión del sonido que tiene ventajas sobre otros, como la sindicación, el almacenamiento y la descarga en dispositivos móviles, solo lo utilizan seis.

\section{Ciencia a demanda}

En la segunda etapa de la investigación, se hace un estudio a detalle de aquellas emisoras donde se cuenta con algún tipo de plataforma para compartir audio con la finalidad de comunicar ciencia y tecnología. Se localizaron 9 emisoras de universidades e institutos públicos y 2 de privadas, que suman 28 producciones, las cuales se muestran en la siguiente lista y a detalle en anexo.

1. "100cia abierta al tiempo"

2. "A ciencia cierta"

3. "Ambiente puma"

4. "Café Tesla"

5. "Cambio climático"

6. "Cápsulas"

7. "Ciencia beat"

8. "Diálogos"

9. "Diálogos del pensamiento"

10. "El árbol de las ideas"

11. "El camino del cangrejo"

12. "El catalejo"

13. "Estudio Darwin"

14. "Gravedad 0" 
15. "Hipócrates 2.0"

16. "Impacto universitario"

17. "Innovadores"

18. "La ciencia que somos"

19. "La fulana"

20. "Las voces de la salud"

21. "Momento económico"

22. "Nuestra huella en el planeta"

23. "Obsesión por el cielo"

24. "Radio con-ciencia"

25. "Sobrerruedas"

26. "Unidos por la química"

27. "Versión radio"

El número de emisoras y producciones en esta posibilidad (14\%) no es el deseable, sin embargo, los programas cuentan con una calidad de producción adecuada, que permite atraer audiencia. Los títulos, por ejemplo, en su mayoría son breves y creativos, buscando llamar la atención del posible oyente. La mayoría de estos tiene una duración entre 20 y 60 minutos, y los formatos más comunes son la revista o magazine en 11 de las producciones, seguido de la entrevista en 7 o la tertulia en 4 casos. Aparecen formatos creativos, como el documental, y los microespacios, donde se utilizan recursos como la dramatización, el cuento y fragmentos sonoros.

La mayor parte de programas (36\%) no presenta un tema específico definido, tiene entre sus objetivos abordar todos los temas de la ciencia al ser de tipo revista. De acuerdo con la clasificación por áreas temáticas que maneja el Conacyt en México, identificamos que los programas temáticos más comunes se refieren a las ingenierías y tecnología, y a las ciencias sociales (figura 2), y aunque en menor medida, también existen interesantes producciones especializadas en las áreas de ciencias de la tierra y la biología.

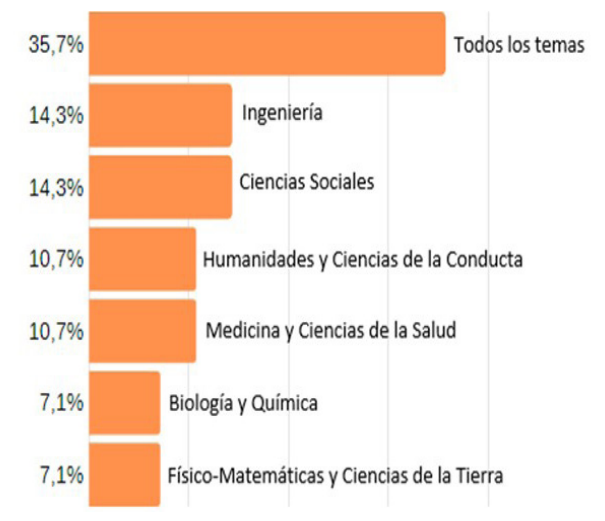

Figura 2. Áreas temáticas de los programas de radio

Fuente: elaboración de la autora. 


\section{DISERTACIONES}

ESTUDIOS

El papel del sonido en la comunicación: contribución, funciones y efectos

ISSN: $1856-9536$

Doi: http://dx.doi.org/10.12804/revistas.urosario.edu.co/disertaciones/a.6550

Volumen 12, Número 2 / Julio-diciembre 2019

Versión PDF para imprimir desde

http://revistas.urosario.edu.co/index.php/disertaciones

Los productores y principales realizadores de estas series radiofónicas son colaboradores o trabajadores de la propia emisora en 11 casos. La comunidad científica tiene también una importante participación, pues 16 de estos productos los elaboran profesores de facultades y de centros de investigación y programas. Entre ellos destacan: el Programa Universitario del Medio Ambiente EcoPuma, el Instituto de Investigaciones Económicas de la unam, el Programa Universitario de Investigación en Salud de la unam, el Programa Universitario de Bioética de la unam o el Departamento de Ecología y Recursos Naturales de la UdeG.

Destaca en este grupo el programa "Estudio Darwin", efectuado por el sindicato de académicos de la Universidad de Guadalajara en Jalisco (STAUdeG) y el programa "Obsesión por el cielo", realizado para la Radio UdeM, y su propio canal de podcast por el Club de Astronomía de la Universidad de Monterrey.

La Universidad Nacional Autónoma de México y la Universidad Autónoma Metropolitana cuentan con departamentos o secciones para la divulgación de la ciencia, con personal especializado; ellos aportan en esta muestra tres programas: "100cia abierta al tiempo", de producción interna, y "El catalejo”, programa de corte juvenil, con una gran producción que aborda un tema general nutrido por sondeos, testimonio de expertos, dramatizaciones y canciones con temas afines, elaborado por personal de la Oficina de Investigación de la Ciencia de la Uam. Esta producción obtuvo el Premio Nacional de Periodismo y Divulgación Científica 2013.

En esta categoría experta también destacan esfuerzos como el programa "La ciencia que somos", una coproducción a nivel Iberoamérica de la Dirección General de Divulgación de la Ciencia de la unam, Radio unam y el Instituto Latinoamericano de la Comunicación Educativa (ILCE), que tiene una periodicidad semanal y duración de hora y media, con un trabajo bien coordinado y la colaboración de investigadores de varios países.

\section{Conclusiones y discusión}

En primer lugar, podemos concluir que, si bien el universo de emisoras universitarias es amplio, el proceso de actualización tecnológica ha sido lento y su incursión a la web 2.0 también. En 2005 se registraba que el $60 \%$ de las emisoras además transmitía por internet (Anuies y Unesco, 2007); para 2009 se calculaba al $70 \%$ (Vázquez Guerrero, 2012) y, si bien hacia 2018 se avanzó un poco más en la transmisión online (75\%), unas cuantas radios siguen emitiendo únicamente por antena o quizá ya optaron por hacerlo por Facebook Time, con eventos o programas específicos, acción a la que es difícil dar seguimiento.

El paso hacia el uso de las redes sociales como Facebook ha sido rápido, pues la mayoría lo tiene y se había registrado en estudio previo (Martín Pena \& Ortiz, 2014), no obstante, el podcast no ha sido totalmente adoptado por las radios mexicanas.

Una de las razones por las que estas emisoras no adoptaron el podcast posiblemente tiene que ver con la falta de conocimiento de la plataforma y sus ventajas, como la sindicación por RSs; sin embargo, es una de las mejores estrategias que se están utilizando en otros países, tanto para la educación como para temas especializados y de minorías, como podría ser la ciencia.

Por otra parte, respecto a la labor de comunicar la ciencia no es suficiente contar con políticas, misiones y principios en las universidades para divulgar ciencia por las radios universitarias cuando existen obstáculos como los que han tenido históricamente muchas de estas radios. Hace falta personal y fondos para emprender la aventura de interesar a una audiencia en temas del acontecer científico que nos permita entender mejor nuestro día a día. Se 


\section{DISERTACIONES}

ESTUDIOS

El papel del sonido en la comunicación: contribución, funciones y efectos

ISSN: $1856-9536$

Doi: http://dx.doi.org/10.12804/revistas.urosario.edu.co/disertaciones/a.6550

Volumen 12, Número 2 / Julio-diciembre 2019

Versión PDF para imprimir desde

http://revistas.urosario.edu.co/index.php/disertaciones

requieren sinergias institucionales donde estén de la mano los gabinetes de comunicación, las áreas de divulgación y los medios universitarios, como se ha probado en otros contextos, por ejemplo, España (Parejo, Martín Pena \& Vivas, 2017), donde se ha podido ver, a pesar del poco tiempo que se viene trabajando en producciones de ciencia y proyectos como el iberoamericano "Semillas de Ciencia" (Parejo, Vivas \& Martín Pena, 2018), que es posible enfocarse en compartir el conocimiento, más allá del beneficio que obtenga la imagen de una institución educativa.

\section{Referencias}

1. (21 de junio de 2017). México es el décimo país más poblado del mundo: onu. Excélsior. Recuperado de http://www.excelsior.com.mx/nacional/2017/06/21/1171150

2. Alcíbar, M. (2015). Comunicación pública de la ciencia y la tecnología: una aproximación crítica a su historia conceptual. Arbor, 191(773):a242. Doi: http://dx.doi.org/10.3989/arbor.2015.773n3012

3. ANUIES \& Unesco. (2007). Los medios de comunicación universitarios en México 2005. CD-ROM.

4. Berlín, I. (2000). El derecho a decir: radios universitarias y educativas en México. Revista Latina de Comunicación Social, 27. Recuperado de http://ull.es/publicaciones/latina/aa2000tma/125/irving.html

5. Calvo Hernando, M. (2006). Arte y ciencia de divulgar el conocimiento. Quito (Ecuador): Ciespal.

6. De Semir, V. (2016). La responsabilidad de los medios de comunicación en la divulgación de las ciencias. Educ Med., 17(Supl 2), 51-55. Recuperado de http://www.elsevier.es/es-revista-educacion-medica-71-linkresolver-la-responsabilidad-los-medios-comunicacion-X1575181316601174

7. Didriksson, A. (2008). Educación superior y sociedad del conocimiento en América Latina y el Caribe, desde la perspectiva de la Conferencia Mundial de la Unesco. En C. Tünnermann (Ed.), La educación superior en América Latina y el Caribe: diez años después de la Conferencia Mundial de 1998. Cali: IESALC, UnesCo-PUJ. Recuperado de www.unesco.org.ve/dmdocuments/.../A_diez_anos_dela_conferencia_mundial.pdf

8. Fayard, P. M. (2003). Punto de vista estratégico sobre la comunicación pública de la ciencia y la tecnología. Quark: Ciencia, Medicina, Comunicación y Cultura, (28). Recuperado de http://quark.prbb.org/28-29/028081. htm

9. Fayard, P. M. (2005). La comunicación pública de la ciencia: hacia la sociedad del conocimiento. México: Dirección General de Divulgación de la Ciencia, unam.

10. Gertrudix, M., \& García García, F. (2011). Topografía de los servicios sonoros en la red social. En M. A. Ortiz Sobrino \& N. López Vidales, Radio 3.0. Una nueva radio para una nueva era. La democratización de los contenidos. Madrid: Fragua.

11. Inegi. (2011). Encuesta sobre la percepción pública de la ciencia y la tecnología. Recuperado de http:// www.beta.inegi.org.mx/proyectos/enchogares/especiales/enpecyt/2011/default.html

12. Instituto Mexicano de la Radio (Imer). (2018). Recuperado de http://www.imer.mx/

13. López Vidales, N. (2011). La radio se transforma: nuevas tecnologías, nuevos hábitos y nuevos perfiles para el medio más cercano. En M. A. Ortiz Sobrino \& N. López Vidales, Radio 3.0. Una nueva radio para una nueva era. La democratización de los contenidos. Madrid: Fragua.

14. Martín Pena, D., \& Ortiz Sobrino, M. A. (Eds.). (2014). Las radios universitarias en América y Europa. Madrid: Fragua. 


\section{DISERTACIONES}

ESTUDIOS

El papel del sonido en la comunicación: contribución, funciones y efectos

ISSN: $1856-9536$

Doi: http://dx.doi.org/10.12804/revistas.urosario.edu.co/disertaciones/a.6550

Volumen 12, Número 2 / Julio-diciembre 2019

Versión PDF para imprimir desde

http://revistas.urosario.edu.co/index.php/disertaciones

15. Muñoz, M. R. (2008). Pertinencia y nuevos roles de la educación superior en la región. En C. Tünnermann (Ed.), La educación superior en América Latina y el Caribe: diez años después de la Conferencia Mundial de 1998. Cali: IESALc, Unesco-pus. Recuperado de www.unesco.org.ve/dmdocuments/.../A_diez_anos_dela_ conferencia_mundial.pdf

16. Orozco, C. E. (2016). Tendencias de la investigación académica internacional en la comunicación pública de la ciencia. En S. Herrera Lima (Coord.), Comunicar ciencia en México: tendencias y narrativas. Guadalajara, México: Iteso.

17. Parametría. (2016). Evaluación del Instituto Mexicano de la Radio 2016. Radioescuchas del ImER. Recuperado de http://www.imer.mx/wp-content/uploads/sites/36/reporte_resultados_evaluacion_imer_2016.pdf

18. Parejo Cuéllar, M., Vivas, A., \& Martín Pena, D. (2018). La divulgación científica en las emisoras universitarias. El caso de la fonoteca del proyecto Semillas de Ciencia. Revista Mediterránea de Comunicación/ Mediterranean Journal of Communication, 9(1), 35-47. Recuperado de https://www.doi.org/10.14198/ MEDCOM2018.9.1.2

19. Parejo, M., Martín Pena, D., \& Vivas, A. (2017). La divulgación científica: estructuras y prácticas en las universidades. Barcelona: Gedisa.

20. Polino, C., \& Castelfranchi, Y. (2017). Consumo informativo sobre ciencia y tecnología. validez y relevancia del índice ıcıc para la medición de la percepción pública. En Ricyt, El estado de la ciencia. Principales indicadores de ciencia y tecnología. Iberoamericanos/interamericanos. 2017. Buenos Aires: Red de Indicadores de Ciencia y Tecnología-Iberoamericana-Interamericana. Recuperado de http://www.ricyt.org/publicaciones

21. Radio Educación. (2018). Recuperado de http://www.radioeducacion.edu.mx/

22. Rodero, E., Vázquez, M., Larrea, O., \& Mas, L. (2014). Sonidos que educan entreteniendo. La radio en la educación 2.0. En J. Rodríguez Terceño (Coord.), Creaciones audiovisuales actuales. Madrid: Accı.

23. Rodríguez, R. (2004). El significado de la extensión universitaria. Ponencia presentada en el IV Encuentro Nacional de Extensión Universitaria, Medellín. Recuperado de https://www.ascun.org.co/foro/iveeu/errodriguez.pdf

24. Slotten, R. H. (2006). Universities, public service experimentation, and the origins of radio broadcasting in the United States, 1900-1920. Historical Journal of Film, Radio and Television, 26, 485-504.

25. Tagüeña, J., Rojas, C., \& Reynoso, E. (junio, 2006). La divulgación de la ciencia en México en el contexto de la América Latina. Ponencia presentada en el I Congreso Iberoamericano de Ciencia, Tecnología, Sociedad e Innovación cts+l, México. Recuperado de www.oei.es/historico/memoriasctsi/simposio/simposio04.pdf

26. Unesco. (2015). Informe de la Unesco sobre la ciencia hacia 2020. Recuperado de http://unesdoc.unesco. org/images/0023/002354/235407s.pdf

27. Vázquez Guerrero, M. (2012). La radio universitaria en México y España. Estudio de la participación y formación de los jóvenes (Tesis doctoral, Universidad Pompeu Fabra). Recuperado de https://repositori.upf. edu/handle/10230/17064

28. Vázquez Guerrero, M., Martín Pena, D., \& Parejo, M. (2015). La divulgación científica a través de la radio universitaria en España y México. Razón y Palabra, 19(3-91), 669-686. 


\section{Anexo. Programas de ciencia analizados}

\begin{tabular}{|c|c|c|c|}
\hline $\mathrm{N}^{\circ}$ & $\begin{array}{l}\text { Emisora, universidad y } \\
\text { ciudad }\end{array}$ & Programa & Página web \\
\hline 1 & $\begin{array}{l}\text { 89.5 Radio Universidad, } \\
\text { Universidad Autónoma de } \\
\text { Querétaro, Querétaro }\end{array}$ & "Unidos por la química" & $\begin{array}{l}\text { http://radio.uaq.mx/index.php/programas/207-unidos-por- } \\
\text { la-quimica }\end{array}$ \\
\hline \multirow{5}{*}{2} & \multirow{5}{*}{$\begin{array}{l}\text { Uam Radio, Universidad } \\
\text { Autónoma de México, } \\
\text { Ciudad de México }\end{array}$} & "El catalejo" & $\begin{array}{l}\text { http://uamradio.uam.mx/index.php/11-programacion/ } \\
\text { series-vigentes/11-el-catalejo }\end{array}$ \\
\hline & & $\begin{array}{l}\text { "100cia abierta al } \\
\text { tiempo" }\end{array}$ & $\begin{array}{l}\text { http://uamradio.uam.mx/index.php/programacion/ } \\
\text { series-vigentes/11-programacion/series-vigentes/7-ciencia- } \\
\text { abierta-al-tiempo }\end{array}$ \\
\hline & & "Ciencia beat" & $\begin{array}{l}\text { http://uamradio.uam.mx/index.php/programacion/series- } \\
\text { historico/12-programacion/series-historico/764-ciencia- } \\
\text { beat }\end{array}$ \\
\hline & & "Innovadores" & $\begin{array}{l}\text { http://uamradio.uam.mx/index.php/programacion/series- } \\
\text { historico/12-programacion/series-historico/10-innovadores }\end{array}$ \\
\hline & & "Versión radio" & $\begin{array}{l}\text { http://uamradio.uam.mx/index.php/programacion/series- } \\
\text { vigentes/11-programacion/series-vigentes/23-version-radio }\end{array}$ \\
\hline 3 & $\begin{array}{l}\text { Radio UdeM, Universidad } \\
\text { de Monterrey, San Pedro } \\
\text { Garza García, Nuevo León }\end{array}$ & "Obsesión por el cielo" & https://obsesionporelcielo.net/ \\
\hline \multirow{8}{*}{4} & \multirow{8}{*}{$\begin{array}{l}\text { Radio unam, Universidad } \\
\text { Nacional Autónoma de } \\
\text { México, Ciudad de México }\end{array}$} & $\begin{array}{l}\text { "Nuestra huella en el } \\
\text { planeta" }\end{array}$ & $\begin{array}{l}\text { http://132.248.61.12/?p=pumateca-audio-Nuestra_huella_ } \\
\text { Residuos }\end{array}$ \\
\hline & & & http://www.radiopodcast.unam.mx/podcast/verserie/33 \\
\hline & & "Momento económico" & $\begin{array}{l}\text { http://www.radio.unam.mx/programa_unam/momento- } \\
\text { economico/ }\end{array}$ \\
\hline & & “Hipócrates 2.0” & $\begin{array}{l}\text { http://www.radio.unam.mx/programa_unam/ } \\
\text { hipocrates-2-0/ }\end{array}$ \\
\hline & & "El árbol de las ideas" & $\begin{array}{l}\text { http://www.radio.unam.mx/programa_unam/arbol-las- } \\
\text { ideas/ }\end{array}$ \\
\hline & & "Ambiente puma" & http://www.radio.unam.mx/album/ambientepuma/ \\
\hline & & "El camino del cangrejo" & http://www.radio.unam.mx/album/caminocangrejo/ \\
\hline & & "La ciencia que somos" & $\begin{array}{l}\text { http://www.radio.unam.mx/programa_unam/la-ciencia- } \\
\text { que-somos/ }\end{array}$ \\
\hline 5 & $\begin{array}{l}\text { UADY Radio, Universidad } \\
\text { Autónoma de Yucatán, } \\
\text { Mérida, Yucatán }\end{array}$ & "Impacto universitario" & http://www.radio.uady.mx/impactouniversitario.html \\
\hline
\end{tabular}




\section{DISERTACIONES}

ESTUDIOS

El papel del sonido en la comunicación: contribución, funciones y efectos

ISSN: 1856-9536

Doi: http://dx.doi.org/10.12804/revistas.urosario.edu.co/disertaciones/a. 6550

Volumen 12, Número 2 / Julio-diciembre 2019

Versión PDF para imprimir desde

http://revistas.urosario.edu.co/index.php/disertaciones

\begin{tabular}{|c|c|c|c|}
\hline No & $\begin{array}{l}\text { Emisora, universidad y } \\
\text { ciudad }\end{array}$ & Programa & Página web \\
\hline 6 & $\begin{array}{l}\text { UdeG Autlán, Universidad } \\
\text { de Guadalajara, Autlán, } \\
\text { Jalisco }\end{array}$ & "A ciencia cierta" & http://udgtv.com/radio-udg-2/autlan/a-ciencia-cierta/ \\
\hline \multirow[t]{2}{*}{7} & \multirow{2}{*}{$\begin{array}{l}\text { UdeG Gdl., Universidad de } \\
\text { Guadalajara, Guadalajara, } \\
\text { Jalisco }\end{array}$} & $\begin{array}{l}\text { "Diálogos del } \\
\text { pensamiento" }\end{array}$ & $\begin{array}{l}\text { http://udgtv.com/radio-udeg/guadalajara/dialogos-del- } \\
\text { pensamiento/ }\end{array}$ \\
\hline & & "Estudio Darwin" & http://udgtv.com/radio-udeg/guadalajara/estudio-darwin/ \\
\hline 8 & $\begin{array}{l}\text { UdeG Vallarta, Universidad } \\
\text { de Guadalajara, Puerto } \\
\text { Vallarta, Jalisco }\end{array}$ & "Cambio climático" & $\begin{array}{l}\text { http://udgtv.com/radio-udg-2/puerto-vallarta/cambio- } \\
\text { climatico/ }\end{array}$ \\
\hline \multirow[t]{2}{*}{9} & \multirow{2}{*}{$\begin{array}{l}\text { Concepto Radial, } \\
\text { Tecnológico de Monterrey, } \\
\text { Ciudad de México }\end{array}$} & "A ciencia cierta" & $\begin{array}{l}\text { http://www.conceptoradial.com/programas/a-ciencia- } \\
\text { cierta/ }\end{array}$ \\
\hline & & "Gravedad 0" & http://www.conceptoradial.com/programas/gravedad-0/ \\
\hline \multirow{5}{*}{10} & \multirow{5}{*}{$\begin{array}{l}\text { Radio uABC, Universidad } \\
\text { Autónoma de Baja } \\
\text { California, Mexicali, Baja } \\
\text { California }\end{array}$} & "Radio con-ciencia" & http://radio.uabc.mx/radioconciencia \\
\hline & & "Café Tesla” & http://radio.uabc.mx/content/cafe-tesla \\
\hline & & "Sobrerruedas" & http://radio.uabc.mx/sobrerruedas \\
\hline & & "Diálogos" & http://radio.uabc.mx/dialogos \\
\hline & & "La fulana" & http://radio.uabc.mx/lafulana \\
\hline 11 & $\begin{array}{l}\text { Radio Tecnológico } \\
\text { de Celaya, Instituto } \\
\text { Tecnológico de Celaya, } \\
\text { Guanajuato }\end{array}$ & "Cápsulas" & http://xeitciencia.itc.mx/ \\
\hline
\end{tabular}

